\title{
On Ruckle's conjecture on accumulation games
}

\author{
Steve Alpern ${ }^{1}$, Robbert Fokkink ${ }^{2}$, Ken Kikuta ${ }^{3}$
}

${ }^{1}$ London School of Economics, Department of Applied Mathematics, Houghton Street, London WC2A 2AE, UK

2 Delft University, Faculty of Electrical Engineering, Mathematics And Information Technology, P.O.Box 5031, 2600 GA Delft, NETHERLANDS

3 Kobe University of Commerce, Department of Management Science, Gakuen-Nishi 8-2-1, Kobe City 651-2197, Japan.

CDAM Research Report 2008-20 SEPTEMBER 2008 


\begin{abstract}
In an accumulation game, the Hider secretly distributes his given total wealth $h$ among $n$ locations, while the Searcher picks $r$ locations and confiscates the material placed there. The Hider wins if what is left at the remaining $n-r$ locations is at least 1; otherwise the Searcher wins. Ruckle's Conjecture says that an optimal Hider strategy is to put an equal amount $h / k$ at $k$ randomly chosen locations, for some $k$. We extend the work of Kikuta and Ruckle by proving the Conjecture for several cases, among others: $r=2$ or $n-2$; $n \leq 7 ; n=2 r-1 ; h<2+1 /(n-r-1)$ and $n \leq 2 r$. The last result uses the Erdos-Ko-Rado theorem. We establish a connection between Ruckle's Conjecture and the difficult Hoeffding problem of bounding tail probabilities of sums of random variables.
\end{abstract}

\title{
1. Introduction
}

Accumulation games, as proposed by Ruckle [16] and by Ruckle and Kikuta $[\mathbf{1 1}$, 17], consider the following problem faced by an individual we call the Hider: He has a certain amount of continuous 'wealth' (food, water, arms, money) which he is forced to secretly stash at a given set of locations, to collect later. In the meantime an opposing Searcher (possibly Nature, in the form of storms or other natural disasters; or perhaps an active pilferer) can choose some of these locations and remove all the hidden the material from them. The Hider wins if what remains after confiscation is sufficient to carry out some task (surviving the winter, insurrection, cornering a market). Kikuta and Ruckle give several logistical applications regarding human behavior. An example not mentioned in the earlier literature is that of the 'scatter hoarder' (e.g. a squirrel) who in the autumn hides food in multiple caches in the hope that enough will remain (after natural disasters or active 'pilferage') to survive the winter. The term scatter hoarder was introduced by Morris [15], who initiated what is now a considerable literature in this area of animal behavior.

To model this problem mathematically, we assume a fixed set of location $\mathcal{N}=$ $\{1, \ldots, n\}$ and a given initial wealth $h$. The Hider may distribute his total wealth $h$ among these locations in any way he chooses. We call his strategic variable $w=\left(w_{1}, \ldots, w_{n}\right)$ a weighting, where $w_{i} \geq 0$ is the amount placed at location $i$. We treat $w$ as a measure on $\mathcal{N}$, so that the feasibility condition is $w(\mathcal{N}) \leq h$. In the case of equality, we call $w$ a partition of $h$, that is $h=w_{1}+\ldots+w_{n}$. The Searcher picks any $r$-subset $I \subset \mathcal{N}$. The Hider wins the game if wealth at the $n-r$ survivng locations $\mathcal{N}-I$ satisfies $w(\mathcal{N}-I) \geq 1$. (The threshold of 1 is a convenient normalization.) Otherwise, the Searcher wins. The parameters $r, n, h$ are all fixed. Interpreting this problem as a (zero-sum, win-lose) game, the value (optimal winning probability of the Hider) and optimal strategies exist by standard 
minimax results [1]. Although in some instances the game formulation is useful, it will generally be more convenient to analyze the problem as a discrete optimization problem, as already demonstrated by Kikuta and Ruckle. They showed that the Hider has an optimal strategy consisting of picking a weighting $w$ and placing the $n$ weights $w_{i}$ randomly on the nodes. The Searcher can pick the set $I$ randomly.

Ruckle has made the following remarkable conjecture.

ConjeCture 1 (Ruckle, [17]). For any parameter values $n, r$ and $h$, it is optimal for the Hider to use $k$ equal positive weights and $n-k$ weights of 0 , for some $k \leq n$.

There is no need for the Hider to use weights greater than 1, since he only needs to retrieve mass 1 . If the Hider uses $k=\lfloor h\rfloor$ equal positive weights, then he may just as well use $k$ unit weights. In this case we say that the Hider uses unit weights. More generally, if the Hider partitions $h$ (that is, if $w(\mathcal{N})=h$ ), then of course the positive weights are all $h / k$, but sometimes it is simpler to use smaller weights. As an example, suppose $n=6, r=4$, and $5 / 2<h<3$. It turns out that it is optimal for the Hider to place five weights of $1 / 2$, and one of 0 . He does not need to use all the material. Here Ruckle's Conjecture holds with $k=5$.

Note that $k$ depends on $h, n, r$. For instance, if $(n-r)(h / n) \geq 1$ then the Hider can guarantee a win by partitioning $h$ into $k=n$ equal parts of $(h / n)$. Kikuta and Ruckle [13] showed that the conjecture holds for $r$ equal to 1 and $n-1$, and gave many examples of other parameter values where it is true. This paper establishes other conditions on the parameters for which the conjecture holds, using the complementary variable $s=n-r$ which describes the size of the set of unsearched locations: $s=2$ or $n-2 ; h<2$ and $n=0$ or $1 \bmod s$; $n \leq 7 ; n=2 s+1 ; h<2+1 /(s-1)$ and $n \geq 2 s$. The last result uses the well known Erdös-Ko-Rado Theorem [6] on the size of 'intersecting families' of $s$-sets. We also establish a more tenuous connection between Ruckle's Conjecture and the difficult Hoeffding Problem [10] of bounding tail probabilities of sums of random variables. For general parameters $n, r, h$, Ruckle's Conjecture remains open.

The accumulation games described above are similar to the 'number hides game' that has been studied in $[\mathbf{3}, \mathbf{1 9}]$. Different types of accumulation games have been studied by Kikuta and Ruckle in [11] and [12]. Recently Alpern and Fokkink [2] have studied a modification of the accumulation game of this paper, in which the Searcher cannot simply search any $r$-set, but is restricted to an arbitrary given family of subsets of $\mathcal{N}$. For example, the Hider may distribute his wealth on the nodes of a graph while the Searcher may remove the wealth from any edge.

\section{Notation}

It is notationally easier to analyze the accumulation game from the complementary point of view, in which the Searcher pure strategy is to state the $s$-set $I \subset \mathcal{N}$ which he leaves unsearched, where $s=n-r$. Thus the accumulation game may be 
described by the Hider (secretly) choosing a weighting $w$ on $\mathcal{N}$ with $w(\mathcal{N}) \leq h$, the Searcher picking an $s$-subset $I$ of $\mathcal{N}$, and the Hider winning (with payoff 1 ) if

$$
w(I) \geq 1 \text {. }
$$

Otherwise the Searcher wins (with payoff 0). The value of the game is thus the winning probability of the Hider, assuming best play on both sides. As observed by Kikuta and Ruckle (and generalized by Alpern and Fokkink in [2]) it is optimal for the Searcher to pick a random $s$-set, and hence the Hider faces an optimization problem: Choose $w$ to maximize the number of $s$-sets $I$ with $w(I) \geq 1$. We say that such a subset $I$ is heavy; otherwise we say that it is light. To summarize, an optimal weighting maximizes the number of heavy sets.

It is useful to restrict the parameter values $n, s, h$ we consider, so as to avoid certain trivial (and exception) cases. Note that if $\frac{s h}{n} \geq 1$ then the Hider can guarantee a win by dividing his material into $n$ equal weights of $h / n$. If $h<1$ then obviously the Hider can never win; if $h \geq 1$ then putting all the weight at a single location makes some sets heavy. So we make the following assumption.

Standing assumption: $\frac{s h}{n}<1$ and $h \geq 1$. So for any optimal weighting, not all edges are heavy and not all edges are light.

The family of all $s$-subsets of $\mathcal{N}=\{1, \ldots, n\}$ is a well known object in combinatorics: it is a hypergraph on $\mathcal{N}$. It is convenient to adopt this terminology and we say that an $s$-subset $I$ of $\mathcal{N}$ is an edge and that the elements of $\mathcal{N}$ are nodes. Often it is convenient to impose a partial ordering on the edges. Given edges $I=\left\{i_{1}, \ldots, i_{s}\right\}$ and $J=\left\{j_{1}, \ldots, j_{n}\right\}$ we say $I \succeq J$ if $i_{k} \geq j_{k}$ for $k=1, \ldots, n$. We assume that weightings are ordered so that $w_{1} \leq \ldots \leq w_{n}$. (Of course the Searcher doesn't know this ordering.) In particular $w(I) \geq w(J)$ if $I \succeq J$. The family of all heavy edges forms a hypergraph and the Hider seeks to maximize the number of edges of this hypergraph. We denote the set of heavy edges containing $i$ as $\mathcal{E}_{i}$ and call its cardinality the degree of $i$, denoted $d_{i}$.

\section{Value of the game}

Often, optimal strategies are hard to find and it is easier to determine, or at least bound, the value of a game. So we turn to the value of the game first and in the proofs in this section, we silently assume that the Hider uses an optimal weighting (whatever it may be). We denote the value of the game by $V(n, s, h)$. It is equal to the maximal number of heavy edges divided by $\left(\begin{array}{l}n \\ s\end{array}\right)$.

Lemma 2. The degree sequence $d_{1} \leq \ldots \leq d_{n}$ is increasing and $d_{1}<d_{n}$. In particular there exists edges $I$ and $J$ that have $s-1$ nodes in common such that $I$ is heavy and $J$ is light.

Proof. Let $w_{1} \leq \ldots \leq w_{n}$ be an optimal weighting. For $0 \leq m \leq n-s$ denote $I_{m}=\{m+1, m+2, \ldots, m+s\}$. By our standing assumption, $I_{0}$ is light 
and $I_{n-s}$ is heavy, so there is a largest $j$ with $I_{j}$ light. Hence $I_{j+1}$ is heavy and $I_{j}$ and $I_{j+1}$ have the $s-1$ nodes $\{j+2, \ldots, j+s\}$ in common.

For the second part, for $k<l$, define $\psi=\psi_{k, l}$ to be the set map that replaces $k$ by $l$ when possible (for sets containing $k$ but not $l$ ) and is the identity otherwise. Since $k<l$ and hence $w_{k} \leq w_{l}, w(\psi(I)) \geq w(I)$ for any set $I$, and thus $\psi$ preserves heaviness. Since $\psi$ gives an injection of $\mathcal{E}_{k}$ into $\mathcal{E}_{l}$, we have $d_{k} \leq d_{l}$. Note that for the $j$ of the first part, the injection $\psi_{j, j+s}: \mathcal{E}_{j} \rightarrow \mathcal{E}_{j+s}$ is not a surjection, as $I_{j+1} \in \mathcal{E}_{j+s}-\psi_{j, j+s}\left(\mathcal{E}_{j}\right)$. Hence $d_{j}<d_{j+s}$, and it follows that $d_{1}<d_{n}$.

Suppose that $d_{i}=d_{j}$ for $j>i$. Then the injection $\mathcal{E}_{i} \rightarrow \mathcal{E}_{j}$ is in fact a bijection. So we can reduce $w_{j}$ to $w_{i}$ without decreasing the number of heavy edges: if $d_{i}=d_{j}$ then we may assume that $w_{i}=w_{j}$. It turns out that Ruckle's conjecture is equivalent to the statement that all the $\leq$ signs in the sequence $d_{1} \leq \ldots \leq d_{n}$ are in fact equalities, except for possibly one inequality (one can show that if the Hider uses only two weights, then it is optimal that one of these weights is zero).

It is convenient to think of the value $V(n, s, h)$ as a tail probability. If $s$ numbers $H_{1}, \ldots, H_{s}$ are sampled without replacement from an optimal weighting $\left\{w_{1}, \ldots, w_{n}\right\}$, then $V(n, s, h)$ is the tail probability:

$$
V(s, n, h)=\mathbb{P}\left(H_{1}+\ldots+H_{s} \geq 1\right) .
$$

In the proof below, we denote the sum of random variables by $S_{s}=H_{1}+\ldots+H_{s}$ or simply by $S$ if the number of samples is clear.

Theorem 3. $V(n, s, h)$ is non-decreasing in $h$, decreasing in $n$ and increasing in $s$.

Proof. The hider need not use all the material, so the value is non-decreasing in $h$. To see that $V$ decreases with $n$, let $\left\{w_{1}, \ldots, w_{n}\right\}$ be an optimal weighting for a value $V$. Note that $\sum d_{i}=s V \cdot\left(\begin{array}{l}n \\ s\end{array}\right)$ so it follows from Lemma 2 that $d_{1}<V \cdot\left(\begin{array}{l}n-1 \\ s-1\end{array}\right)$. The number of heavy edges that does not contain the first node is $V \cdot\left(\begin{array}{l}n \\ s\end{array}\right)-d_{1}>$ $V \cdot\left(\begin{array}{c}n-1 \\ s\end{array}\right)$. Hence the weighting $\left\{w_{2}, \ldots, w_{n}\right\}$ yields a value $>V$ and we conclude that $V\left(n-1, s, h-w_{1}\right)>V(n, s, h)$. Since we have established monotonicity in $h$ we have $V(n-1, s, h) \geq V\left(n-1, s, h-w_{1}\right)>V(n, s, h)$ as claimed.

To see that the value increases with $s$, we use that $V(n, s, h)$ is the tail probability $\mathbb{P}\left(S_{s} \geq 1\right)$ for an optimal partition. We sample once more to get $V(n, s+1, h)=$ $\mathbb{P}\left(S_{s+1} \geq 1\right)$. Let $\mathcal{H}$ be the event that $S_{s} \geq 1$, where $S_{s}$ is a sum of random variables. Then

$$
\mathbb{P}\left(S_{s+1} \geq 1\right)=\mathbb{P}\left(S_{s+1} \geq 1 \mid \mathcal{H}\right) \cdot V+\mathbb{P}\left(S_{s+1} \geq 1 \mid \mathcal{H}^{c}\right) \cdot(1-V),
$$

where $V=V(n, s, h)$. Since $\mathbb{P}\left(S_{s+1} \geq 1 \mid \mathcal{H}\right)=1$ it suffices to show that $\mathbb{P}\left(S_{s+1} \geq\right.$ $\left.1 \mid \mathcal{H}^{c}\right)>0$. In other words, it suffices to show that there exists a light edge that can be made heavy by adding just one node. This is the content of Lemma 2 . 


\section{THEOREM 4.}

$$
1-e^{-\frac{s\lfloor h\rfloor}{n}} \leq V(n, s, h) \leq \frac{\lfloor s h\rfloor}{n}
$$

The proof of this theorem borrows ideas from the study of the accumulation game on the circle, that has been carried out in $[\mathbf{2}]$.

Proof. We calculate modulo $n$ and we think of $\{1, \ldots, n\}$ as a circle. We say that a subset $I_{j}=\{j+1, \ldots, j+s\}$ is an $s$-interval. Notice that $\sum w\left(I_{j}\right)=s h$ and that there are $n$ intervals. Suppose that the Searcher adopts a restricted strategy and randomly picks an $s$-interval. The value of this 'restricted game' is greater then or equal to $V(n, s, h)$ since a restriction of the Searcher benefits the Hider. The sum of random variables $S=H_{j+1}+\ldots+H_{j+s}$ has expectation $\mathbb{E}[S]=\frac{s h}{n}$. It follows from Markov's inequality $\mathbb{P}(S \geq 1) \leq \mathbb{E}[S]=\frac{s h}{n}$. There are $n$ intervals, so at most $\lfloor s h\rfloor$ of them can be heavy. Therefore the value of the restricted game is at most $\frac{\lfloor s h\rfloor}{n}$.

The lower bound follows from the Searcher strategy of placing $\lfloor h\rfloor$ unit weights. The number of heavy edges is $\left(\begin{array}{l}n \\ s\end{array}\right)-\left(\begin{array}{c}n-\lfloor h\rfloor \\ s\end{array}\right)$ in this case and if we divide this by the total number of edges $\left(\begin{array}{l}n \\ s\end{array}\right)$ we obtain the lower bound $1-\prod_{i=0}^{s-1}\left(1-\frac{\lfloor h\rfloor}{n-i}\right)$ on $V(n, s, h)$. The theorem now follows from the fact that $\prod_{i=0}^{s-1}\left(1-\frac{\lfloor h\rfloor}{n-i}\right) \leq$ $\left(1-\frac{\lfloor h\rfloor}{n}\right)^{s} \leq e^{-\frac{s\lfloor h\rfloor}{n}}$.

It is tempting to try another probability inequality to bound the value of the game. The Azuma-Hoeffding inequality for sums of independent random variables, which was first proved in $[\mathbf{1 0}$, Thm 1], also applies to random samples without replacement [10, Thm 4], but unfortunately, it gives no better bound then Markov's inequality in our case.

If $\frac{s\lfloor h\rfloor}{n}$ is small, then the lower bound in Theorem 4 is $\frac{s\lfloor h\rfloor}{n}$ up to order $O\left(\left(\frac{s\lfloor h\rfloor}{n}\right)^{2}\right)$. Surprisingly, $\frac{s\lfloor h\rfloor}{n}$ is an absolute upper bound under some arithmetic restrictions on $s$ and $n$ :

ThEOREM 5. If $n=0 \bmod s$ or $n=1 \bmod s$, then $V(n, s, h) \leq \frac{s\lfloor h\rfloor}{n}$.

Proof. Again, as in the proof of the previous theorem, the Searcher adopts the restricted strategy of taking one of these $s$-intervals at random, uniformly. The value of the restricted game is bounded by $\frac{\lfloor s h\rfloor}{n}$ and by our standing assumption $s h<n$ so there exists a light interval. Without loss of generality we may assume that $I_{n-1}$ is light (note that we cannot assume that $w_{1} \leq \ldots \leq w_{n}$ since the Hider adopts a restricted strategy).

To prove the theorem, it suffices to show that there are at most $s\lfloor h\rfloor$ heavy $s$ intervals. Since $n=0,1 \bmod 1$ there exist an integer $k$ such that either $n=k s$ or 
$n=k s+1$. Since $I_{0} \cup I_{s} \cup \ldots \cup I_{(k-1) s}$ is a disjoint union, the sum of the weights of these intervals is at most $h$. So at most $\lfloor h\rfloor$ of these intervals can be heavy. The same argument applies to $I_{j} \cup I_{s+j} \cup \ldots \cup I_{(k-1) s+j}$ and we find that at most $s\lfloor h\rfloor$ of the intervals $I_{j+i s}$ are heavy with $0 \leq j<s$ and $0 \leq i<k$. For the given restrictions on $j$ and $i$ we find all intervals except $I_{n-1}$ for $n=k s+1$. But $I_{n-1}$ is light.

The arithmetic restriction on $s$ and $n$ is necessary. If $n=5$ and $s=3$ and $h=3 / 2$ then the Hider divides into $\left\{\frac{1}{2}, \frac{1}{2}, \frac{1}{2}, 0,0\right\}$, creating 7 heavy $s$-sets. It follows from Theorem 16 below that this weighting is optimal. The value of the game is $V(5,3,3 / 2)=\frac{7}{10}$, while $\frac{s\lfloor h\rfloor}{n}=\frac{3}{5}$ and $\frac{\lfloor s h\rfloor}{n}=\frac{4}{5}$.

The following corollary will be improved later on.

COROllary 6. If $h<2$ and if $n=0$ or $1 \bmod s$ then the Hider uses a single unit weight. In particular, Ruckle's conjecture holds.

Proof. Since $\lfloor h\rfloor=1$ the lower bound $1-\prod_{i=0}^{s-1}\left(1-\frac{\lfloor h\rfloor}{n-i}\right)$ in the proof of Theorem 4 is equal to $\frac{s}{n}=\frac{s\lfloor h\rfloor}{n}$. By Theorem 5 this is the value of the game.

COROLlaRY 7. If $n=0 \bmod s$ and if $h \geq \frac{n-1}{s}$ then Ruckle's conjecture is true.

Proof. Under these conditions $\lfloor h\rfloor=\frac{n-s}{s}$. By Theorem 5 the number of light edges is at least $\frac{n-s\lfloor h\rfloor}{n} \cdot\left(\begin{array}{l}n \\ s\end{array}\right)=\left(\begin{array}{c}n-1 \\ s-1\end{array}\right)$. Now suppose the Hider puts $n-1$ weights $\frac{1}{s}$. Then the number of light edges is $\left(\begin{array}{c}n-1 \\ s-1\end{array}\right)$ which is best possible.

These corollaries are typical for the results in our paper. We can only prove the conjecture if $\frac{s\lfloor h\rfloor}{n}$ is close to 1 or if it is relatively small.

Ruckle's conjecture is true if $s=1$ or $n-1$, see [13]. In the next section, the conjecture is shown to be true as well if $s=2$ or $n-2$. This suggests that there exists some a symmetry between sampling $s$ times or $n-s$ times in an accumulation game. We can prove that such a symmetry exists but only under a very restrictive condition on the optimal weighting:

THEOREM 8. Suppose that there exists an optimal weighting with weights bounded by $\frac{h-1}{n-s-1}$, for $s<n-1$ and $h>1$. Then $V(n, s, h)=V\left(n, n-s, \frac{h(s+1)-n}{h-1}\right)$.

Proof. Let $w=\left\{w_{1}, \ldots, w_{n}\right\}$ be an optimal weighting of mass $h$. Define a new weighting $w^{\prime}$ with weights $g_{i}=1-\left(\frac{n-s-1}{h-1}\right) \cdot w_{i}$, which is well defined under the conditions, and total weight $g=\frac{(h(s+1)-n}{h-1}$. Conversely, any such weighting $w^{\prime}$ can be transformed to a weighting $w$ with weights $\leq \frac{n-s-1}{h-1}$ by the inverse transformation $w_{i}=\frac{1-g_{i}}{n+1-s-g}$. Now compute

$$
w_{1}+\ldots+w_{s} \geq 1 \Leftrightarrow w_{s+1}+\ldots+w_{n} \leq h-1 \Leftrightarrow
$$




$$
\begin{gathered}
\frac{n-s-1}{h-1} \cdot w_{s+1}+\ldots+\frac{n-s-1}{h-1} \cdot w_{n} \leq n-s-1 \Leftrightarrow \\
\left(1-g_{s+1}\right)+\ldots+\left(1-g_{n}\right) \leq n-s-1 \Leftrightarrow g_{s+1}+\ldots+g_{n} \geq 1 .
\end{gathered}
$$

In particular, an edge is heavy $w(I) \geq 1$ under the weighting $w$ if and only if its complement is heavy $w^{\prime}\left(I^{c}\right) \geq 1$ under the weighting $w^{\prime}$.

\section{Solution of the conjecture for some special cases}

It is not hard to see that Ruckle's conjecture is true if $s=1$ or $s=n-1$. If $s=1$ then the Hider divides $h$ into $\lfloor h\rfloor$ unit weights (or more precisely, into $\lfloor h\rfloor$ parts of weight $h /\lfloor h\rfloor$ ). If $s=n-1$ then the hider puts one weight $h$. In this section we prove that Ruckle's conjecture is also true if $s=2$ or $s=n-2$.

Lemma 9. For any partition $h=w_{1}+\ldots+w_{n}$ there exists another partition $g=g_{1}+\ldots+g_{n}$ for $g \leq h$ and all $g_{i} \in\left\{0, \frac{1}{2}, 1\right\}$, such that $w_{i}+w_{j} \geq 1$ implies $g_{i}+g_{j} \geq 1$.

The proof of this lemma is similar to one used for arbitrary graphs in $[\mathbf{2}]$.

Proof. Let $\mathfrak{R}$ be the set of all equations $w_{i}+w_{j} \geq 1$ that are satisfied by a weighting $\left\{w_{1}, \ldots, w_{n}\right\}$. Without loss of generality, we may assume that $h$ minimizes $w_{1}+\ldots+w_{n}$ under the constraints $\mathfrak{R}$ and $w_{i} \geq 0$. Denote $b=$ $\max \left\{w_{i}: w_{i} \neq 1\right\}$ and $a=\min \left\{w_{i}: w_{i} \neq 0\right\}$, so that $a \leq b$. Call the weights $w_{i}$ which are equal to $b$ the 'big weights' and those equal to $a$ the 'small weights'.

If $a+b>1$ then $h$ could be reduced by decreasing all the big weights to $1-a$, contradicting our assumption that $h$ is minimal under the constraints $\Re$. Similarly, if $a+b<1$ then we could decrease all the small weights to zero. Hence $a+b=1$.

Let $\alpha$ be the number of small weights, let $\beta$ be the number of big weights, and let $\varepsilon$ be the minimum difference between any two weights. If $\beta>\alpha$ we could decrease $h$ by changing the big weights to $b-\varepsilon$ and increasing the small weights to $a+\varepsilon$, contrary to assumption. If $\beta<\alpha$, then $h$ may be reduced by changing the small weights to $a-\varepsilon$ and the big ones to $\beta+\varepsilon$. We conclude that $\alpha=\beta$.

Minimize $|b-a|$ under the constraints $\mathfrak{R}$ and $h=w_{1}+\ldots+w_{n}$ and $w_{i} \geq 0$. We claim that $|b-a|=0$. If not, then we could reduce the big weights to $b-\varepsilon$ and increase the small weights to $a+\varepsilon$ under the constraints, since $\alpha=\beta$, contradicting minimality. We conclude that $a=b=\frac{1}{2}$ and we are done.

The proof of the following lemma is very similar.

LEMma 10. For any partition $h=w_{1}+\ldots+w_{n}$ there exists another partition $g=g_{1}+\ldots+g_{n}$ with $g \geq h$ such that $g_{i}+g_{j}<g-1$ if $w_{i}+w_{j}<h-1$ and such that of the following two possibilities holds:

(1) all $g_{i} \in\left\{0, \frac{h-1}{2}, h-1\right\}$

(2) $g$ is trivially partitioned into $g+0+\ldots+0$. 


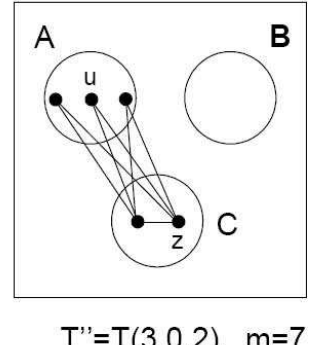

$T^{\prime \prime}=T(3,0,2), \quad m=7$
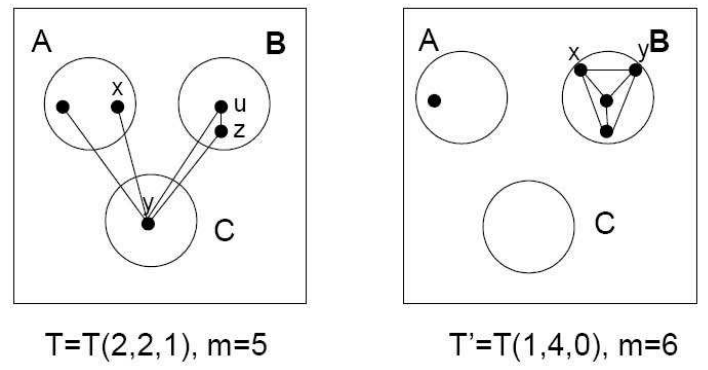

Figure 1. mass $m$ of a $T$-graph

Proof. Suppose that the weighting contains a weight $>h-1$, say $w_{1}$. Then the trivial partition $h+0 \ldots+0$ satisfies the same constraints $w_{i}+w_{j}<h-1$ and the second possibility holds. So suppose that all weights are $\leq h-1$. Let $b=\max \left\{w_{i}: w_{i} \neq h-1\right\}$ and $a=\min \left\{w_{i}: w_{i} \neq 0\right\}$, so that $a \leq b$. Let $\mathfrak{R}$ be the set of all equations $w_{i}+w_{j}$ that are satisfied by the weights of the partition. We minimize $h$ under these constraints.

If $a+b>h-1$ then $h$ could be increased by replacing all the big weights by $h-1$. Similarly, if $a+b<h-1$ then $h$ could be increased by replacing the small weights by $m-1-b$. Hence $a+b=h-1$. Let $\alpha$ be the number of small weights, let $\beta$ be the number of big weights. If $\beta<\alpha$ we could reduce $b$ to $b-\varepsilon$ and increase $a$ to $a+\varepsilon$, contrary to our choice of a maximal $h$. In the same way we can rule out that $\beta>\alpha$. We conclude that $\alpha=\beta$. Minimize $|b-a|$ under the constraints $\mathfrak{R}$ and $h=w_{1}+\ldots+w_{n}$ and $w_{i} \geq 0$. By the same argument as in the previous lemma $|b-a|=0$. We conclude that $a=b=\frac{h-1}{2}$.

In the proofs of both lemmas we treated the equations $w_{i}+w_{j}$ as constraints. We never needed that $i$ and $j$ are different, so the lemmas remain valid if we allow that $i=j$.

Definition 11. A graph is called a T-graph if its nodes can be partitioned into three sets $A, B$ and $C$ such that a pair of nodes is connected if and only of one of the nodes is in $C$ or if both nodes are in $B$. If the cardinalities of the three node sets are respectively $a, b$ and $c$, we denote the graph by $T(a, b, c)$, and we define its mass to be $b+2 c$.

Lemma 12. Let $T(a, b, c)$ be a $T$-graph with $a, c \geq 1$ and $b \geq 2$. Then there is another T-graph with the same nodes and the same mass which has more arcs than $T$.

Proof. We consider two overlapping cases, $b \geq a$ and $b \leq a$.

$b \geq a$ : Since $a$ and $c$ are at least 1 , we may move two nodes $x$ and $y$ from $A$ and $C$, respectively, to $B$. There will be $b$ more edges incident to $x$, 
corresponding to the original nodes of $B$, and $a-1$ fewer edges incident to $y$. Hence there are $b-(a-1) \geq 1$ more edges in the resulting $T$-graph. $b \leq a$ : Since $b \geq 2$, we may move two nodes $u$ and $z$ from $B$ into $A$ and $C$, respectively. In the resulting $T$-graph there will be $b-1$ fewer edges incident to $u$, corresponding to the nodes of $B$ other than $u$ and $z$, and $a$ more edges incident to $z$. Hence the resulting $T$-graph has $a-(b-2) \geq 1$ more edges.

THEOREM 13. Ruckle's conjecture is true if $s=2$ or $s=n-2$.

Proof. Suppose that $s=2$. The optimal weighting maximizes the number of $w_{i}+w_{j} \geq 1$ and by Lemma 9 we may suppose that the weights are either 0 or $\frac{1}{2}$ or 1 . Hence, the equations $w_{i}+w_{j} \geq 1$ form the edges on a $T$-graph, with $A$ the set of zero weights, $B$ the set of weights $\frac{1}{2}$ and $C$ the set of unit weights. The optimal weighting maximizes the number of edges of the $T$-graph. By Lemma 12 either $b \leq 1$, or $\min \{a, b\}=0$. If $b=1$ there is only one weight $\frac{1}{2}$, which could be changed to a zero weight without losing optimality. So if $b \leq 1$ then $b=0$ and the Hider uses zero and unit weights only, in accordance with the conjecture. If $a=0$ then the all $w_{i}+w_{j} \geq 1$ contradicting our standing assumption. So $c=0$ and the Hider uses only weights 0 and $\frac{1}{2}$ in this case, in accordance with the conjecture.

Suppose that $s=n-2$. The optimal weighting minimizes the number of $w_{i}+w_{j}<$ $h-1$ and by Lemma 10 there are two cases. The case of the trivial weighting $\{h, 0, \ldots, 0\}$ is in accordance with the conjecture. So we may suppose that the weights are either 0 or $\frac{h-1}{2}$ or $h-1$. The equations $w_{i}+w_{j}<h-1$ form the edges of a $T$-graph, with $A$ the set of weights $h-1, B$ the set of weights $\frac{h-1}{2}$ and $C$ the set of zero weights. By a similar analysis, which we leave to the reader, we conclude that either $a$ or $b$ is zero.

COROllary 14. Ruckle's conjecture is true for $n \leq 6$.

Proof. Since the conjecture is correct if $s \in\{1,2, n-2, n-1\}$ it is true for $n \leq 5$. For $n=6$ the remaining case is $s=3$, which is settled by corollary 6 .

The following theorem will be extended in the next section.

TheOREM 15. If $n=2 s+1$ then Ruckle's conjecture is true.

Proof. If $h<2$ then Corollary 6 applies. Suppose that $h \geq 2$ and note that $h<2+\frac{1}{s}$ by our standing assumption. If the Hider puts two unit weights then he creates $\left(\begin{array}{c}2 s+1 \\ s\end{array}\right)-\left(\begin{array}{c}2 s-1 \\ s\end{array}\right)$ heavy edges. This is optimal if the number of light edges is always at least $\left(\begin{array}{c}2 s-1 \\ s\end{array}\right)$. Since $\left(\begin{array}{c}2 s-1 \\ s\end{array}\right)=\frac{1}{2}\left(\begin{array}{c}2 s \\ s\end{array}\right)$ it suffices to show that of the edges with nodes in $\{2, \ldots, 2 s+1\}$ half are light. Suppose that $I$ and $J$ are complementary edges in $\{2, \ldots, 2 s+1\}$ and suppose that both are heavy. Then $s w_{1} \geq w(I) \geq 1$ so $w_{1} \geq \frac{1}{s}$ and therefore $h \geq w_{1}+w(I)+w(J) \geq 2+\frac{1}{s}$ contradicting our standing 
assumption. It follows that of each pair of complementary edges at least one is light. So half of the edges with nodes in $\{2, \ldots, 2 s+1\}$ are light.

By symmetry one would expect to dispose of the case $n=2 s-1$ in a similar manner, but this is not entirely true.

TheOREm 16. If $n=2 s-1$ and $h \geq 2-\frac{1}{s-1}$ then Ruckle's conjecture is true.

Proof. Suppose $h \geq 2-\frac{1}{s-1}$. We show that the partition into $2 s-3$ weights $\frac{1}{s-1}$ is optimal. This creates $\left(\begin{array}{c}2 s-3 \\ s-2\end{array}\right)$ light edges. Let $w_{1}+\ldots+w_{n}$ be any partition of $h$ with $w_{1} \leq \ldots \leq w_{n}$. Note that $w_{k}<\frac{1}{s}$ otherwise all edges are heavy. We say that two edges $I, J$ are 1 -complementary if $\{1\}=I \cap J$. Two such edges cannot both be heavy since $w(I)+w(J)=h+w_{1}$ which is $<2$ by our standing assumption. So the number of light edges is at least $\frac{1}{2}\left(\begin{array}{c}2 s-2 \\ s-1\end{array}\right)=\left(\begin{array}{c}2 s-3 \\ s-2\end{array}\right)$.

We illustrate below for $s=7$ and $n=4$ that the case $h<2-\frac{1}{s-1}$ is more involved.

COROLlary 17. Ruckle's conjecture is true for $n=7$.

Proof. The cases $s \in\{1,2,5,6\}$ are settled by the preceding theorem and $s=3$ is settled by Theorem 15. It remains to settle the case $s=4$. By Theorem 16 we may assume that $h<5 / 3$. We show that an optimal weighting in this case is $\{0,0,0,0,1 / 2,1 / 2,1 / 2\}$, which gives 22 heavy edges. So we need to argue that there are at least 13 light edges, which we do by contradiction and assume that there are less. Since $I=\{2,3,4,7\}$ has 12 edges that are smaller in the $\succ$ order, it has to be heavy. We argue that its 3 -complementary edge $J=\{1,3,5,6\}$ is heavy. Indeed, if $I$ and $J$ they would both be heavy, then $h+w_{3}=w(I)+w(J) \geq 2$. So $w_{3} \geq 1 / 3$ but this is nonsense since this implies that $h \geq 5 / 3$. In particular, at most one of $I=\{2,3,4,7\}$ and $J=\{1,3,5,6\}$ is heavy and since $I$ is heavy, $J$ is light. There are 8 edges that are smaller than $J$ in the edge order, so they are light also. The edges $\{2,3,5,6\}$ and $\{1,3,4,7\}$ are 3 -complementary, so at least one of the following cases holds:

(A) $\{2,3,5,6\}$ is light

(B) $\{1,3,4,7\}$ is light

Assume that (A) holds. Since $\{2,3,5,6\}$ is larger than 11 edges, we need just one more light edge to get a contradiction. The edges $\{1,2,3,7\}$ and $\{2,4,5,6\}$ are 2-complementary, so at least one of them is light. This gives 13 light edges, contradicting our assumption that there are at most 12. Assume that (B) holds. There are two edges that are smaller than $\{1,3,4,7\}$ and that are not in the set of 9 light edges that are $\preceq J$ for a total of 12 light edges. The edge $\{2,3,4,6\}$ is not in this set so it is heavy by our assumption. Its 3-complementary edge $\{1,3,5,7\}$ therefore is light and this is the 13-d edge that is light. We conclude that $\{0,0,0,0,1 / 2,1 / 2,1 / 2\}$ is an optimal weighting if $h<5 / 3$. 
Now suppose that $h<3 / 2$. We claim that it is optimal to put one unit weight, for a total of 20 heavy edges. We argue by contradiction and assume that there are less than 15 edges that are light. This implies that the three edges $\{3,4,5,6\},\{2,3,5,7\},\{1,4,5,7\}$ all are heavy, since each has 14 edges that are smaller. By the familiar argument, only one of two 5-complementary edges can be heavy. So the three edges $\{1,2,5,7\},\{1,4,5,6\},\{2,3,5,6\}$ are light, but there are 13 edges that are smaller than one of these three edges, contradicting our assumption.

A weaker form of Ruckle's conjecture is that the Hider uses at least $n-s h$ zero weights in an optimal partition. The following result is a step toward settling this weakened conjecture.

THEOREM 18. For any $n, s, h$ there exists an optimal weighting for the Hider with at least $n-s^{2}\lfloor h\rfloor$ zero weights.

Proof. Fix some optimal weighting and let $\left\{I_{1}, \ldots, I_{k}\right\}$ be a maximal family of disjoint heavy edges, so that $k \leq\lfloor h\rfloor$, and let $\mathcal{I}=I_{1} \cup \ldots \cup I_{k}$. Every heavy edge contains at least one node in $\overline{\mathcal{I}}$. Let $\mathcal{J}=\left\{j: w_{j}>0\right\}$ be the nodes of weight $>0$ in the hider's partition. Suppose that $|\mathcal{J}|>s^{2}\lfloor h\rfloor$. Let $\epsilon$ denote the minimum nonzero weight. Reduce the weight on the nodes that are in $\mathcal{J} \backslash \mathcal{I}$ by $\epsilon$ and increase the weight on the nodes in $\mathcal{I}$ by $(s-1) \epsilon$. There are more than $s^{2}\lfloor h\rfloor-s\lfloor h\rfloor$ nodes in $\mathcal{J} \backslash \mathcal{I}$ and there are at most $s\lfloor h\rfloor$ nodes in $\mathcal{I}$, so this operation does not increase the total weight of the partition. It preserves heavy sets, since each heavy set contains a node that increases by $(s-1) \epsilon$. So we can reduce the total weight until $\mathcal{J}$ contains no more then $s^{2}\lfloor h\rfloor$ nodes.

\section{Intersecting families}

Let $\mathcal{F}$ be a family of subsets of $\{1, \ldots, n\}$. In other words, $\mathcal{F}$ is a hypergraph. It is called an intersecting family if no two of its elements are disjoint.

TheOREM 19 (Erdös-Ko-Rado, [6]). Let $\mathcal{F}$ be an intersecting family of s-subsets. If $2 s \leq n$ then $\mathcal{F}$ has no more than $\left(\begin{array}{c}n-1 \\ s-1\end{array}\right)$ elements. In other words, the family of sets with one common element has maximal cardinality.

The following improves on Corollary 6 and Theorem 15 .

Corollary 20. Ruckle's conjecture is true if $h<2+\frac{1}{s-1}$ and $n \geq 2 s$.

Proof. If $h<2$ then the heavy edges form an intersecting family and by the Erdös-Ko-Rado theorem the Hider puts one unit weight. Consider the case that $h \geq 2$. Suppose that $w_{n}<\frac{1}{s-1}$. Then all weights are smaller than $\frac{1}{s-1}$ so each heavy edge consists of $s$ weights $\geq \frac{1}{s}$. It follows that the Hider may just as well use weights $\frac{1}{s}$ only, which is a strategy that is predicted by Ruckle's conjecture. So we may assume that $w_{n} \geq \frac{1}{s-1}$. Now $h-w_{n}<2$, so the family of heavy edges that do not contain node $n$ form an intersecting family $\mathcal{F}$. By the Erdös-Ko-Rado 
theorem $|\mathcal{F}| \leq\left(\begin{array}{c}n-2 \\ s-1\end{array}\right)$. There are $\left(\begin{array}{c}n-1 \\ s-1\end{array}\right)$ edges that contain node $n$, so the number of heavy edges is bounded by

$$
\left(\begin{array}{c}
n-2 \\
s-1
\end{array}\right)+\left(\begin{array}{c}
n-1 \\
s-1
\end{array}\right)=\left(\begin{array}{c}
n \\
s
\end{array}\right)-\left(\begin{array}{c}
n-2 \\
s
\end{array}\right)
$$

which is the number of heavy edges if the Hider puts two unit weights.

The Erdös-Ko-Rado theorem is a celebrated result and a starting point of the theory of hypergraphs [4]. It has been extended in many ways. For integers $n, s, k$ the number $f(n, s, k)$ is defined as the largest possible collection of $s$-sets, no $k$ of which are pairwise disjoint, that can be chosen from a set of size $n .{ }^{1}$ Obviously, if $k=\lfloor h\rfloor$ then the maximal number of heavy edges is bounded by $f(n, s, k)$.

TheOrem 21 (Erdös, [7]). For each $s \geq 2$ there exists a constant $c(s)$ depending only on s such that

$$
f(n, s, k)=\left(\begin{array}{l}
n \\
s
\end{array}\right)-\left(\begin{array}{c}
n-k+1 \\
s
\end{array}\right) \text { for } n>c(s) k .
$$

The value of $f(n, s, k)$ in this theorem is attained by the family of all $s$-subsets that contain at least one element of a given $k-1$-subset. In other words, it is attained if the Hider uses unit weights:

COROLlary 22. Ruckle's conjecture is true if $n>c(s)\lfloor h\rfloor$ and in this case the Hider uses $\lfloor h\rfloor$ unit weights.

The best known estimate of the constant in Theorem 21, due to Bollobás et al [5], is $c(s) \leq 2 s^{3}$.

\section{Hoeffding's problem}

Ruckle's conjecture is related to work of Hoeffding and others in probability. Suppose that the Searcher samples randomly and with replacement, so he may pick the same weight twice. Unlike in Ruckle's accumulation game, it is not easy to give a real life interpretation of this game, but it does simplify the random variables. In particular, the samples $X_{1}, \ldots, X_{s}$ now are independent and the Hider wants to maximize the tail probability $\mathbb{P}\left(X_{1}+\ldots+X_{s} \geq 1\right)$ for i.i.d. random variables. This is related to a probability problem that was proposed by Hoeffding [8] and studied by Hoeffding and Shrikhande [9]. Hoeffding's problem is to find nonnegative i.i.d. random variables that maximize $\mathbb{P}\left(X_{1}+\ldots+X_{s} \geq 1\right)$ for a given $\mathbb{E}\left[X_{i}\right]=\alpha$.

TheOrEm 23 (Hoeffding-Shrikhande). If $s=2$ and if $2 \alpha<1$ then the tail probability is maximized by either $X_{i} \in\left\{0, \frac{1}{2}\right\}$ or $X_{i} \in\{0,1\}$.

\footnotetext{
${ }^{1}$ This number is also denoted by $f(n, s, k, 0)$, where 0 represents empty intersection and Erdös in [7] denotes it by $f(n, s, k)-1$. In other papers, the number $f(n, s, k)$ represents the maximum cardinality of a union of $k$ intersecting families.
} 
Note that the random variable $X_{i}$ is well defined, since it takes only two values and since its expectation is known. The Hoeffding-Shrikhande theorem is similar to our Theorem 13.

Hoeffding's problem has been proposed in several contexts, as discussed in [14]. In Meester's words, the problem satisfies a common rule: $s=1$ is trivial, $s=2$ can be solved with a reasonable amount of work and $s \geq 3$ is hard. There is no conjectured solution to Hoeffding's problem, but the general idea seems to be that the tail probability can be maximized by a random variable that takes on only two values. The only result on Hoeffding's problem apart from the HoeffdingShrikhande theorem is the following asymptotic result.

Theorem 24 (Samuels [18]). Let $X_{i}$ be i.i.d. and non-negative for $1 \leq i \leq s$. If $\max \{4 s h / n,(s-1) s h / n\}<1$ then the tail probability is maximized by $X_{i} \in\{0,1\}$.

In particular, if $2 s^{2} h<n$ and if $h$ is an integer, then a weighting by unit weights is optimal. Note the similarity with our Corollary 22 and also note that the order $s^{2}$ is sharper then $s^{3}$ as found by Bollobás et al.

Hoeffding's problem is not exactly the same as the problem of finding an optimal weighting in an accumulation game with replacement. For instance, if $s=2$ and $n=4$ and $h=3 / 2$ then $\mathbb{E}\left[X_{i}\right]=3 / 8$. By the Hoeffding-Shrikhande theorem the tail probability is maximized by random variables $X_{i} \in\{0,1\}$ (which give a greater tail probability under these conditions then $X_{i} \in\left\{0, \frac{1}{2}\right\}$ ). However, these random variables cannot be created by a weighting on 4 locations. The optimal weighting is $\left\{0,0, \frac{1}{2}, 1\right\}$. Suppose we double the number of locations $n=8$ and the mass $h=3$, keeping the expectation at $\mathbb{E}\left[X_{i}\right]=3 / 8$, then it is possible to create the optimal random variables by the weighting $\{0,0,0,0,0,1,1,1\}$, which therefore is optimal. Any solution of Hoeffding's problem puts an upper bound on the value of the accumulation game with replacement.

\section{Acknowledgement}

We would like to thank Graham Brightwell for pointing out that the Erdös-KoRado theorem settles Ruckle's conjecture if $h<2$ and $n \geq 2 s$ and that Erdös' theorem settles the conjecture if $n$ is sufficiently large. 


\section{Bibliography}

[1] S. Alpern and S. Gal (1988), A Mixed Strategy Minimax Theorem without Compactness, SIAM J. Control Optimiz., Vol. 26, 1357-1361

[2] S. Alpern and R.J. Fokkink (2008), Accumulation games on graphs, preprint.

[3] V. J. Baston, F. A. Bostock and T. S. Ferguson (1989), The number hides game, Proc. Amer. Math. Soc. 107, No. 2 , pp. 437-447

[4] B. Bollobas (1986), Combinatorics, Cambridge University Press, Cambridge, 1986.

[5] B. Bollobas, D.E. Daykin, P. Erdös (1976), Sets of independent edges of a hypergraph, Quart. J. Math. Oxford (2), 21, 25-32.

[6] P. Erdös, C. Ko, R. Rado (1961), Intersection theorems for systems of finite sets, Quart. J. Math. Oxford Ser. (2) 12, 313-320.

[7] P. Erdös (1965), A problem on independent r-tuples, Ann. Univ. Sci. Budapest, Eötvös Sect. Math. 8, 93-95.

[8] W. Hoeffding (1955), The extrema of the expected value of a function of independent random variables, Ann. Math. Stat. 26, 268-275.

[9] W. Hoeffding, S.S. Shrikhande (1955), Bounds for the distribution function of a sum of independent, identically distributed random variables, Ann. Math. Stat. 26, 439-449.

[10] W. Hoeffding (1963), Sums of independent random variables, J. Amer. Stat. Soc. 58, 13-30.

[11] K. Kikuta, W. Ruckle (1997), Accumulation games, Part 1: noisy search, J. Optimiz. Th. Appl. 94, no. 2, 395-408.

[12] K. Kikuta, W. Ruckle (2000), Continuous accumulation games in continuous regions, J. Optimiz. Th. Appl. 106, no. 3, 581-601.

[13] K. Kikuta, W. Ruckle (2001), Continuous accumulation games on discrete locations, Naval Res. Logist. 41, no. 6, 821-831.

[14] L.E. Meester (2008), Extremal distributions for tail probabilities of sums of iid random variables on [0,1], preprint, Arxive 0808.1669v1.

[15] D. Morris (1962), The behaviour of the green acouchi (Myoprocta pratti) with special reference to scatter hoarding, Proc Zool. Soc. Lond. 139, 701-732

[16] W. Ruckle (1983), Geometric games and their applications, Pitman Research Notes in Mathematics 82, Boston.

[17] W. Ruckle (2001), Accumulation games, Sci. Math. Jpn. 54, no. 1, 173-203.

[18] S.M. Samuels (1969), The Markov inequality for sums of independent random variables, Ann. Math. Stat. 40, 1980-1984.

[19] N. Zoroa, M.J. Fernández-Sáez, P. Zoroa (1999), A game related to the number of hides game, J. Optimiz. Th. Appl. 103, 457-473. 